\title{
4. SNAPSHOT OF ADULT HEALTH, NSW, 2003
}

S(Smoking) N(Nutrition and Obesity) A(Alcohol) P(Physical Activity) S(Psychological Distress) H(Health Status and Health Services) $\mathbf{O}$ (Oral Health, Asthma and Diabetes) $\mathbf{T}$ (Trust and Social Capital)

\section{SNAPSHOT OF ADULT HEALTH, NSW, 2003}

\begin{tabular}{|c|c|c|c|c|c|}
\hline Topic & Issue & Indicator & $\begin{array}{c}\text { Male } \\
(\%)\end{array}$ & $\begin{array}{c}\text { Female } \\
(\%)\end{array}$ & $\begin{array}{c}\text { Persons } \\
(\%)\end{array}$ \\
\hline \multirow[t]{6}{*}{ Health behaviours } & Alcohol & Alcohol risk drinking & 41.5 & 30.4 & 35.9 \\
\hline & Fruit & Recommended daily fruit intake & 39.0 & 52.5 & 45.9 \\
\hline & Vegetables & Recommended vegetable intake & 11.8 & 26.7 & 19.3 \\
\hline & Physical Activity & Adequate physical activity & 49.7 & 40.7 & 45.1 \\
\hline & Smoking & Current daily or occasional smoking & 24.9 & 20.0 & 22.4 \\
\hline & Smoke free households & Smoke-free households & - & - & 82.6 \\
\hline \multirow[t]{6}{*}{ Health status } & Health status & Excellent, very good, or good self-rated health status & 81.9 & 79.8 & 80.8 \\
\hline & Asthma & Current asthma & 9.2 & 12.7 & 11.0 \\
\hline & Diabetes & Diabetes or high blood sugar & 6.9 & 5.5 & 6.2 \\
\hline & Psychological distress & High and very high psychological distress & 9.3 & 12.9 & 11.1 \\
\hline & Oral Health & All natural teeth missing & 4.2 & 7.4 & 5.8 \\
\hline & Overweight or obesity & Overweight or obesity & 55.6 & 41.0 & 48.3 \\
\hline \multirow[t]{3}{*}{ Health services } & $\begin{array}{l}\text { Difficulty getting health } \\
\text { care }\end{array}$ & Difficulties getting health care when needing it & 11.4 & 15.2 & 13.3 \\
\hline & $\begin{array}{l}\text { Emergency department } \\
\text { care rating }\end{array}$ & $\begin{array}{l}\text { Emergency department care rated as excellent, } \\
\text { very good, or good }\end{array}$ & 80.2 & 77.7 & 79.0 \\
\hline & Hospital care rating & Hospital care rated as excellent, very good, or good & 93.0 & 89.8 & 91.2 \\
\hline \multirow[t]{4}{*}{ Social capital } & Participation & $\begin{array}{l}\text { Attended a community event at least once in the } \\
\text { last six months }\end{array}$ & 54.5 & 62.2 & 58.4 \\
\hline & Trust & Most people can be trusted & 71.6 & 68.0 & 69.8 \\
\hline & Safety & Feel safe walking down their street after dark & 80.4 & 56.8 & 68.4 \\
\hline & $\begin{array}{l}\text { Reciprocity-Social } \\
\text { engagement }\end{array}$ & Visit neighbours & 66.9 & 64.0 & 65.4 \\
\hline
\end{tabular}

